\title{
Severe combined immunodeficiency (SCID) associated neutropenia: a lesson from monozygotic twins
}

Tim Niehues, Klaus Schwarz, Marion Schneider, Horst Schroten, Elisabeth Schröder, Volker Stephan, Volker Wahn

\begin{abstract}
A case of severe combined immunodeficiency (SCID) in monozygotic twin sisters was detected at 3 months of age with neutropenia in one twin and a normal differential count in the other. The neutropenic twin, suffering from severe skin ulcers, was successfully treated with granulocyte colony stimulating factor (GCSF). Discordant occurrence of neutropenia in identical twins shows that there may be a non-genetic cause for the neutropenia in SCID. Suppression of myelopoiesis was probably induced by activated maternal $T$ cells. The neutropenia in this case may thus be classified as SCID associated neutropenia, as opposed to reticular dysgenesis, in which the neutropenia is G-CSF refractory and is most probably caused by a genetic stem cell defect. A response to G-CSF in a neutropenic child with SCID can be clinically beneficial and might help to distinguish between G-CSF unresponsive reticular dysgenesis and G-CSF responsive SCID associated neutropenia.

(Arch Dis Child 1996; 74: 340-342)
\end{abstract}

Keywords: severe combined immunodeficiency (SCID), neutropenia, granulocyte colony stimulating factor (G-CSF).

University of Düsseldorf, Düsseldorf, Germany

Department of

Paediatrics

T Niehues

H Schroten

Stephan

V Wahn

Department of Transfusion Medicine M Schneider

Department of Human Genetics

E Schröder

University of Ulm, Ulm, Germany

Department of Paediatrics II K Schwarz Correspondence to:
Prof Dr med Volker Wahn, Prof Dr med Zentrum für Kinderheilkunde der Heinrich Heine Universitä Düsseldorf, Moorenstr 5,

Accepted 12 December 1995
We had the unique opportunity to study two genetically identical individuals with SCID Only one twin presented with severe neutropenia, showing that there is an initial presentation of neutropenia in SCID which has a non-genetic aetiology and requires specific initial management.

\section{Case reports}

CASE 1

Twin I was referred to our clinic at the age of 3 months with a history of fever, diarrhoea, and deep skin ulcerations; Pseudomonas aeruginosa and enterococci were detectable on culture. After an apparently normal pregnancy the girl and her twin sister (case 2) had been born to healthy, consanguineous Turkish parents. A 2 year old sister is healthy. On admission twin 1 was in a good general condition with a weight of $5500 \mathrm{~g}$ (25th centile for Turkish girls). Physical examination revealed several deep skin ulcerations on both thighs each at least $2 \times 2 \mathrm{~cm}$ in size. A complete blood count showed a decreased haemoglobin level $(7.0 \mathrm{~g} / \mathrm{dl})$ and packed cell volume $(22 \%)$, a normal platelet count $(326000 / \mu \mathrm{l})$ and 1600 leucocytes/ $\mu \mathrm{l}$ with a differential blood count of $77 \%$ lymphocytes, $7 \%$ monocytes, $10 \%$ banded cells, and $6 \%$ polymorphonuclear neutrophils (absolute neutrophil count: $256 / \mu 1$ ). Cord blood results were not available. Antigranulocyte antibodies were not detected. The bone marrow showed a developmental arrest at the stage of promyelocytes. Because the girl developed chronic rotavirus infection, $B$ and $T$ cell function was evaluated. Levels of immunoglobulins were low (IgG $<91 \mathrm{mg} / \mathrm{dl}$; IgM $<14 \mathrm{mg} / \mathrm{dl} ;$ IgA $<8 \mathrm{mg} / \mathrm{dl}$ ). Mature $\mathrm{T}$ cells were markedly decreased, with an increased relative percentage of $B$ cells. The complete surface marker profile is given in the table. Peripheral blood B and T cells were separated immunomagnetically and $\mathrm{T}$ cells were identified as being of maternal origin by HLA typing. The response of peripheral blood lymphocytes to mitogens was markedly decreased (table). The thymus could barely be detected by sonography. Adenosine deaminase and purine nucleoside phosphorylase levels were normal. Severe B and T cell dysfunction led to the diagnosis of SCID.

The skin infections and ulcers barely responded to different intravenous antibiotic regimens. Therefore, $1 \mu \mathrm{g} / \mathrm{kg}$ of granulocyte colony stimulating factor (G-CSF) (Neupogen, Amgen, Munich) was given subcutaneously on alternate days. A response was noted on day 6 and neutrophil counts above $2000 / \mu l$ could be maintained. Ulcers completely healed within two weeks. After withdrawal of G-CSF, neutrophil counts again fell 
Immunological studies. Surface marker profile and mitogen response. Numbers indicate percentages of total peripheral blood lymphocytes staining with each marker by flow cytometry or counts per minute (cpm) in the thymidine incorporation assay

\begin{tabular}{lccr}
\hline & Twin I & Twin II & Control \\
\hline Tand B cell markers & & & \\
CD3+ & 30 & 19 & 87 \\
CD4+ & 13 & 1 & 58 \\
CD8+ & 12 & 11 & 25 \\
CD20+ & 51 & 67 & 6 \\
NK cell markers & & & \\
CD56/CD3- & 6 & 1 & 5 \\
Adhesion markers & & & \\
CD2+ & 35 & 19 & 92 \\
CD11a & 94 & 97 & 97 \\
CD18 & 96 & 96 & 100 \\
Activation marker/HLA & expression & & \\
CD4/HLA-DR+ & 11 & 0 & 0 \\
CD8/HLA-DR+ & 8 & 10 & 100 \\
HLA-ABC & 99 & 95 & \\
Mitogen response (cpm) & & & \\
Medium alone & 470 & 200 & 120 \\
PHA & 1380 & 1750 & 33940 \\
OKT3 & 210 & 920 & 24000 \\
PWM & 1700 & 160 & 31320 \\
SAC & 2100 & 390 & 4820 \\
\hline
\end{tabular}

PHA = phytohaemagglutinin: $\mathrm{PWM}=$ pokeweed mitogen; $\mathrm{SAC}=$ staphylococcal protein $\mathrm{A}$ of Cowan I strain.

below $500 / \mu 1$ within five days. As dependence on G-CSF was documented, treatment was continued until bone marrow transplantation.

Desquamative exanthema developed on hands, feet, face, and skull. As this was associated with a rise in transaminases it was felt most likely to be the result of graft versus host $(\mathrm{GvH})$ reaction caused by maternal $\mathrm{T}$ cells. During more than two months of hospital inpatient care before bone marrow transplantation our patient had severe failure to thrive. After the decision to carry out a bone marrow transplant had been made, a central venous catheter was inserted and parenteral nutrition given.

\section{CASE 2}

Twin II was referred to our clinic one day after her twin sister for treatment of pneumonia. On admission she weighed $4500 \mathrm{~g}$ (below 10th centile for turkish girls) but was normal on physical examination. Laboratory investigations on admission were also normal except for a moderately increased $\gamma$ glutamyl transferase and an increased leucocyte count $(24300 / \mu 1$ with a normal differential count), as well as a slightly raised $C$ reactive protein $(1.8 \mathrm{mg} / \mathrm{dl})$. Pneumonia was confirmed by chest $x$ ray. After treatment with intravenous antibiotics she made a rapid recovery from the pneumonia. When the diagnosis of SCID was established in twin I we also evaluated twin II for possible immunodeficiency. Genetic identity with twin I was confirmed by DNA fingerprinting in granulocytes (data not shown), as desribed by Wong et al. ${ }^{4}$ Using eight microsatellites, the calculated probability of monozygosity was $99.95 \%$ according to the Bayes principle of conditional probabilities. Laboratory examination revealed a very similar immunological phenotype (table).

In the course of the disease patient 2 also acquired chronic rotavirus gastroenterits, an exanthema most probably caused by $\mathrm{GvH}$ reaction, and severe failure to thrive requiring parenteral nutrition.

\section{Discussion}

We have described genetically identical twins with different phenotypes of SCID. Both twins displayed clinical as well as laboratory findings consistent with SCID. The most surprising finding was that only one twin was severely neutropenic at presentation, although genetic identity was demonstrated by DNA fingerprinting. A possible alloimmune aetiology for the neutropenia was evaluated but no antigranulocytic antibodies were detected. Cellular immune mechanisms involving maternal $\mathrm{T}$ cells were not further analysed. An infectious cause could not entirely be excluded but seemed unlikely because neutropenia recurred when G-CSF was discontinued, at a time when there was no clinical or laboratory evidence of infection.

It is well known that materno-fetal transfusion of $\mathrm{T}$ cells can be demonstrated in more than 50 per cent of patients with SCID. ${ }^{5} \mathrm{We}$ believe that suppression of myelopoeisis in our case was most probably explained by a GvH reaction caused by maternal $T$ cells. As maternal $T$ cells were found in both children, it seems likely that there were major differences in the effector functions of maternal $T$ cells between the twins. In the neutropenic twin I a much stronger activation of $T$ cells (percentage of HLA-DR+ $T$ cells, see the table) was observed. There was indirect evidence that haematopoetic suppression also involved erythropoiesis, as routine evaluation of the anaemia did not reveal any other cause, and the anaemia persisted, with haemoglobin concentrations between 6 and $8 \mathrm{~g} / \mathrm{dl}$.

In the original report describing reticular dysgenesis it was stated that there may be several grades of severity. Severe cases of neutropenia in SCID resembling the original report usually present at birth or in the first week, with almost no detectable neutrophils; these cases are fatal within days to weeks. ${ }^{167}$ Attempts to treat a severe case and her sister using G-CSF $(4-30 \mu \mathrm{g} / \mathrm{kg})$ failed. A genetic stem cell defect seems likely in these cases because myelopoiesis was resistant to G-CSF in vivo as well as in the absence of maternal $\mathrm{T}$ cells in vitro. ${ }^{7}$

In the most recent report of reticular dysgenesis, treatment with granulocyte macrophage colony stimulating factor (GM-CSF) resulted in reconstitution of normal neutrophil counts. ${ }^{3}$ A difference in stem cell sensitivity to G-CSF or GM-CSF might explain the divergent response. However, a different severity of neutropenia in SCID may account for the reported difference. Less severe forms of SCID and neutropenia, including the GM-CSF responsive case and our own, had most of the following features in common: a late manifestation (one to three months), a less profound neutropenia at presentation $(>100$ neutrophils $/ \mathrm{ml}$ ), and a better overall prognosis. ${ }^{38}$ Passively transferred immunity and breast feeding might have prevented an earlier onset 
of symptoms. ${ }^{8}$ Aetiologically a GvH induced mechanism seems likely in these less severe cases. Both a primary stem cell defect and suppression of haematopoiesis by maternal $T$ cells are compatible with the finding of normal neutrophil numbers in SCID following bone marrow transplantation. ${ }^{8}$ Thus treatment of the defect is either linked to reconstitution with a normal stem cell/haematopoietic system or to elimination of maternal $\mathrm{T}$ cells in the process of conditioning for bone marrow transplantation.

Ownby et al suggested the descriptive term 'SCID with leucopenia' instead of reticular dysgenesis. ${ }^{2}$ Now that haematopoietic growth factors are in clinical use, it is useful to differentiate between SCID associated neutropenia, which is less severe and potentially responsive to treatment with GM-CSF/G-CSF, and the more severe reticular dysgenesis, which appears to be refractory to G-CSF. Administration of G-CSF in a child presenting with SCID and severe neutropenia should differentiate between these two conditions.
We thank Dr G Kögler (Bone Marrow Donor Centre, Düsseldorf, Germany) for separation and HLA-typing of maternal T cells, Prof $\mathrm{K}$ Welte (Department of Paediatrics, Hannover, Germany) for discussion and comments on bone marrow morphology, Prof C Müller-Eckardt (Department of Paediatrics, Giessen, Germany) for analysis of antigranulocytic antibodies, and Dr U Schauer (Department of Paediatrics, Bochum, Germany) for investigation of the adenosine deaminase and purine nucleoside phosphorylase levels.

1 De Vaal OM, Seynhaeve V. Reticular dysgenesis. Lancet 1959; ii: 1123-5.

2 Ownby DR, Pizzo S, Blackmon L, Gall SA, Buckley RH. Severe combined immunodeficiency with leukopenia (reticular dysgenesis) in siblings: immunologic and histopathologic findings. $\mathcal{F}$ Pediatr 1976; 89: 382-7.

3 Azcona C, Alzina V, Barona P, Sierrasesumaga L, VillaElizaga I. Use of recombinant human granulocyteElizaga 1. Use of recombinant human granulocytemacrophage colony stimulating factor in an infant

4 Wong Z, Wilson V, Patel I, Povey S, Jeffreys AJ. CharacWong Z, Wilson V, Patel I, Povey S, Jeffreys AJ. Charac-
terization of a panel of highly variable minisatellites cloned from human DNA. Ann Hum Genet 1987; 51: 269-88.

5 Stephan JL, Vlekova V, Le Deist F, et al. Severe combined immunodeficiency: a retrospective single-center study of clinical presentation and outcome in 117 patients. $\mathcal{F}$ Pediatr 1993; 123: 564-72.

6 Roper M, Parmley RT, Crist WM, Kelly DR, Cooper MD Severe congenital leukopenia (reticular dysgenesis). $A m \mathcal{F}$ Dis Child 1985; 139: 832-5.

7 Bujan W, Ferster A, Sariban E, Friedrich W. Effect of recombinant human granulocyte colony-stimulating factor in reticular dysgenesis (letter). Blood 1993; 82: 1684.

8 Levinsky RJ, Tiedeman K. Successful bone-marrow transplantation for reticular dysgenesis. Lancet 1983; i: 671-3.
inst 